\title{
New Measuring Technique for Reducing the Number of Voltage Sensors in Modular Multilevel Converters
}

\author{
Ricard Picas, Student Member, IEEE, Jordi Zaragoza, Member, IEEE, Josep Pou, Senior Member, IEEE, \\ Salvador Ceballos, and Josep Balcells, Senior Member, IEEE
}

\begin{abstract}
This paper presents a new technique for measuring the capacitor voltages in a modular multilevel converter (MMC) using a reduced number of voltage sensors. With this technique, the minimum number of voltage sensors per arm is two. Each sensor measures the output voltage of a set of submodules (SMs) connected in series and acquires a new measurement when there is only one SM activated within the set. The acquired value corresponds to the capacitor voltage of the activated SM minus the voltage drops produced in the switches. A simple mathematical model is used to estimate all the SM capacitor voltages, and it is then updated whenever there is a new measurement available. An algorithm that enforces the periodic update of the voltage measurements is also presented. The proposed measuring technique highly reduces the number of voltage sensors, hence reducing the complexity and costs of the signal conditioning and data acquisition stages. Simulation and experimental results are presented to demonstrate the efficiency of the proposed technique.
\end{abstract}

Index Terms-Modular multilevel converter, Voltage measurement, Capacitor voltage estimator, Reduced number of sensors.

\section{INTRODUCTION}

$\mathbf{M}$ ULTILEVEL converters are attractive power converter topologies for medium and high power applications [1], [2]. Among the multilevel converter topologies, the modular multilevel converter (MMC) [3]-[7] offers several salient features which make it a competitive solution for high-voltage direct current (HVDC) transmission systems [8], [9] and flexible alternating current transmission systems (FACTS) [10]. The most attractive features of the MMC are [6]: (i) its modularity and scalability to different power and voltage levels, (ii) its high efficiency, (iii) the high quality of the output voltages, and (iv) the absence of additional capacitors on the

This work was supported by the Ministerio de Economía y Competitividad of Spain under Project ENE2012-36871-C02-00, partially funded by the European Union, and the Consolider-Ingenio Program under Project CD200900046.

R. Picas, J. Zaragoza and J. Balcells are with the Terrassa Industrial Electronics Group, Technical University of Catalonia, 08222 Terrassa, Spain (e-mail: ricard.picas@upc.edu; jordi.zaragoza-bertomeu@upc.edu; josep.balcells@upc.edu).

J. Pou is with the Australian Energy Research Institute and the Schoo of Electrical Engineering and Telecommunications, The University of New South Wales, Sydney, NSW 2052, Australia, on leave from the Technical University of Catalonia, 08034 Barcelona, Spain (e-mail: j.pou@unsw.edu.au; josep.pou@upc.edu).

S. Ceballos is with the Energy Unit, Tecnalia Research and Innovation, 48160 Derio, Spain (e-mail: salvador.ceballos@tecnalia.com). dc-link, as the storage is distributed among the capacitors in the submodules (SMs) of the converter.

The general topology of an MMC consists of two arms per phase-leg, where each arm comprises $N$ series-connected, identical SMs and a series arm inductor, $L$. Each SM consists of a half-bridge circuit and a capacitor $C$. The output voltage of each SM $\left(v_{S M}\right)$ is either equal to its capacitor voltage when the SM is activated $\left(v_{C}\right)$, or it is zero when deactivated. To synthesize the voltage waveforms at the ac-side of the MMC, multiple modulation techniques can be used [6]. Most of them are based on defining the number of SMs to be activated in each of the arms, and the particular SMs are determined by a voltage balancing algorithm [11], [12]. Almost all of these voltage balancing techniques operate in closed-loop and hence the values of the SM capacitor voltages need to be known. In this paper, a phase-disposition PWM (PD-PWM) technique with a voltage balancing algorithm based on sorting the capacitor voltages is used.

The capacitor voltages are usually obtained by direct measurement of all the SM capacitors. In real applications of the $\mathrm{MMC}$, the number of SMs per arm $(N)$ can reach into the hundreds [8]. Therefore, a large number of voltage sensors is required in order to provide signal measurements that need to be acquired and adapted. Aside from compromising its reliability, this complicates the implementation and control of the MMC.

Several studies have been performed to solve this challenge. Multiple open-loop techniques, based on modulation patterns or carrier rotation, have been developed [13]-[15], but their stability may be compromised because of the open-loop operation. Recently, techniques based on advanced estimators have also appeared. In [16], the capacitor voltage values are calculated from other variables including the arm currents and the dc-link voltage, and in [17], the capacitor voltages are estimated by the arm currents and corrected by measuring the arm voltages. Closed-loop voltage balancing algorithms that reduce the processing time in MMCs with a large number of SMs have also been proposed [11], [18], [19], however they do not reduce the number of voltage sensors required. A technique to reduce the hardware complexity of the voltage sensing stage maintaining closed-loop operation was presented in [20]. In that publication, the SM capacitor voltage signals are multiplexed, measuring only a part of the signals each sampling period. The SM capacitor voltages are estimated in between the measurements. Although the technique presented 


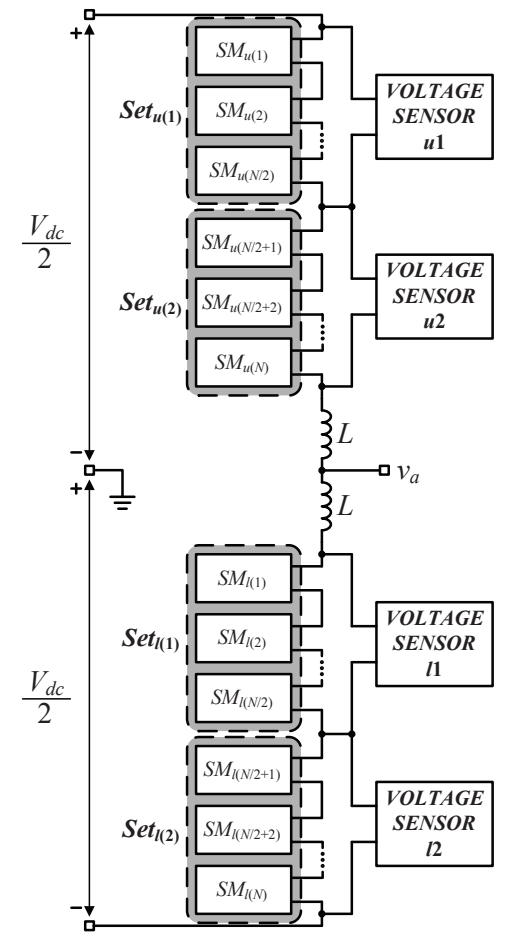

Fig. 1. Distribution example of measuring sensors on a phase-leg. Each arm is divided into two sets of SMs.

in [20] does not reduce the number of sensors, it highly reduces the complexity of the acquisition stage.

In this paper, a new technique to determine the SM capacitor voltages by using a reduced number of voltage sensors is presented. The minimum number of voltage sensors required per arm is two. Each voltage sensor is connected at the output of a set of SMs in series, acquiring the voltage measurement only when one SM of the set is activated. The acquired value corresponds to the capacitor voltage of the activated SM minus the voltage drops in the semiconductors, which can be easily estimated. Similarly to [20], since the measurements of all the capacitor voltages are not always available, they are estimated by a mathematical model between actual measures. In this mathematical model the capacitor voltage values are updated whenever there is an actual measurement available. In order to ensure a periodic update of each of the estimated capacitor voltages, an algorithm that enforces measurement of each capacitor voltage is also introduced to improve the proposed sensing technique.

The proposed measuring technique can be implemented instead of the traditional individual measurement technique but using significantly fewer voltage sensors. This technique is capable of estimating the capacitor voltages properly under both steady-state operation and transients. However, the measuring system might not be able to operate properly if one or more SMs fail. The proposed technique can also be used as a complementary measuring system in order to improve reliability by providing the system with fault tolerance. This is a topic that is being extensively studied nowadays [21]-[24]. Providing the measuring system with fault-tolerance capability by using the proposed technique will be studied in future

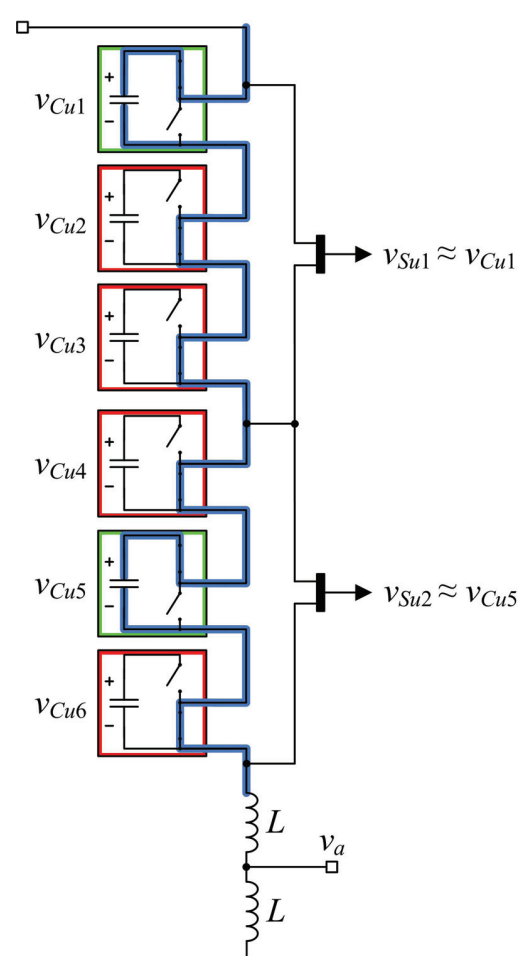

Fig. 2. Measurement example in an arm with six SMs $(N=6)$.

research work.

The remainder of this paper is organized as follows. Section II defines the operating principles of the measuring technique. Section III describes the enforced measuring algorithm. Section IV present simulation and experimental results. Section $\mathrm{V}$ summarizes the main conclusions of this work.

\section{PRINCIPLES OF OPERATION}

\section{A. Voltage Measurement}

In the proposed measuring technique, each arm of the converter is divided into sets of SMs, using a voltage sensor for each set. The sensors are connected at the output of the seriesconnected SMs, measuring the sum of the voltages supplied by the SMs of the specific set. The measured voltage $v_{S j x}$, where $j$ indicates the upper or lower $\operatorname{arm}(j=\{u, l\})$ and $x$ the number of the measuring set, is a multilevel waveform that will be acquired when only one SM of the measuring set is activated, obtaining a voltage almost equal to the capacitor voltage of the activated SM. The difference between the real and the measured capacitor voltage corresponds to the voltage drop in the on-state switches, which can be considered negligible or be estimated easily.

In order to avoid switching noise, the voltage measurements are synchronized with the pulse-width modulator. The capacitor voltage is sampled at the midpoint between switchings, i.e. when the triangular carrier is maximum and minimum. Moreover, the measurements acquired very close to a switching transition are rejected.

Fig. 1 shows a diagram of a phase-leg of the converter dividing each arm into two sets. The figure shows the distribution 


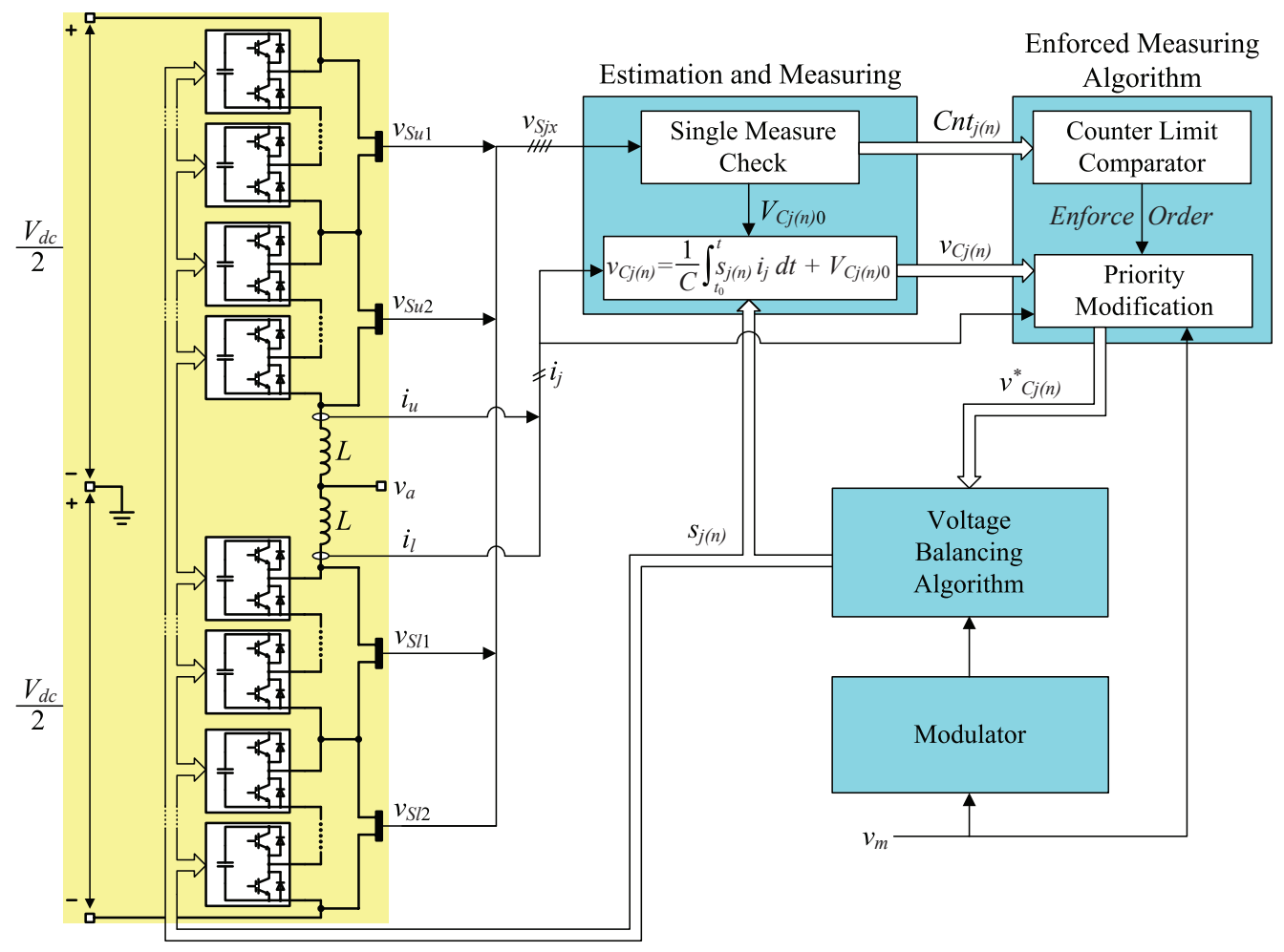

Fig. 3. Block diagram of the proposed measuring technique.

of the sets and the connection points of the sensors in the phase-leg.

A measurement example of an MMC arm with $N=6$ is depicted in Fig. 2. Since there is only one SM activated in each set at this particular instant, each sensor in the example measures the voltage of a single SM capacitor; i.e., $v_{S u 1}$ and $v_{S u 2}$ are practically equal to $v_{C u 1}$ and $v_{C u 5}$, respectively.

\section{B. Voltage Estimation}

Due to its principle of operation, the measuring technique on its own does not provide the instantaneous capacitor voltages at all times. Therefore, it is complemented with an algorithm that calculates the estimated capacitor voltage value since the last measurement. The estimation has to be performed for all the SMs, so a simple and fast-processing algorithm should be used. By knowing the state of the SMs $s_{j(n)}$ and the arm current $i_{j}$, the capacitor voltages $v_{C j(n)}$ are calculated by:

$$
v_{C j(n)}=\frac{1}{C} \int_{t_{0}}^{t} s_{j(n)} i_{j} d t+V_{C j(n) 0},
$$

where $C$ is the nominal value of the capacitors, $n=\{1, \ldots, N\}$ indicates the number of $\mathrm{SM}, t_{0}$ is the last measuring time of the capacitor voltage, and $V_{C j(n) 0}$ is the measured capacitor voltage at $t_{0}$. When a new measurement of the capacitor voltage is available, the integral term in (1) is reset and $V_{C j(n) 0}$ becomes the measured voltage. Hence, the error accumulated in the capacitor voltage estimator is periodically eliminated by incorporating the actual voltage measurement.

The use of estimation algorithms that are executed continuously in the central processing unit (CPU), allows responding rapidly to dynamic changes of the converter operation. Any standard CPU can process the estimation algorithm very quickly, allowing the MMC controllers to operate with no practical speed limitations due to the implementation of the proposed measuring technique. The periodic measurements of the capacitor voltages are used only to update the values of the estimators in order to avoid accumulative errors in the estimated voltages.

In fact, in MMCs with a high number of levels, the proposed technique can even reduce the response time because the estimation algorithm can be executed faster than the acquisition of many SM capacitor voltages.

In this paper, the proposed measuring technique includes a simple and fast-processing algorithm for the estimation of the capacitor voltages. However, more accurate estimation models, like those presented in [16] and [20], could also be used.

\section{System Integration}

The block diagram depicted in Fig. 3 shows the different processes that integrate the proposed voltage measuring technique. First, the set voltages $\left(v_{S j x}\right)$ are acquired and their correspondence with a single capacitor voltage measurement is checked. An individual counter $\left(C n t_{j(n)}\right)$ is used for each SM and it is increased with any new measurement. If the measure corresponds to a single SM capacitor voltage, that value is updated in the estimator and its corresponding counter is reset. The estimated voltages are sent to the enforced measuring algorithm that, if necessary, modifies their values in order to facilitate the single measurement of a particular capacitor. The performance of this algorithm is detailed in Section III. 
As shown in the block diagram in Fig. 3, the measuring technique provides the capacitor voltages to the voltage balancing algorithm as an input, without affecting its internal performance. Therefore, this technique is not only independent of the modulation technique implemented, but also on the algorithm used to balance the capacitor voltages. In the case of using a voltage balancing algorithm without switching frequency limiter, estimation inaccuracies may modify the switching frequency because several SMs can be activated and deactivated at any switching transition to balance the capacitor voltages. However, if a voltage balancing algorithm with controlled switching frequency is used [25], [26], only one SM is activated or deactivated per switching transition, achieving constant switching frequency regardless the value of the capacitor voltages.

The proposed technique reduces significantly the number of voltage sensors required in the MMC. It provides input information continuously to the voltage balancing algorithm and consequently its processing time is not affected. Further investigation can lead to new voltage balancing algorithms with reduced processing times thanks to using the knowledge of the capacitor voltages dynamics to avoid sorting all the SMs every sampling period.

\section{Minimum Number of Sensors}

The measuring technique could be applied by dividing each arm into only one measuring set of SMs; i.e., using one voltage sensor per arm. However, its application range would be limited, since voltage measurements would be performed when only one SM per arm is activated. In MMCs with a high number of SMs, this situation would never happen when operating with low modulation indices. For this reason, the measuring technique is used with a minimum of two sensors per arm, each one of them will measure the voltage supplied by half of SMs in the arm. The use of two measuring sets per arm allows measurements to be performed when $N / 2+1$ SMs or less are activated, a situation that appears for all the modulation indices. To perform the measurement, only one SM in a measuring set is activated and the rest are in the other set. Furthermore, although the minimum number of sensors in the arm is two, this number can be increased in order to improve the measuring frequency and therefore reduce the maximum error accumulated in the capacitor voltage estimators.

An example of the operation range of the proposed technique depending on the number of sets per arm is depicted in Fig. 4. In this example, the number of SMs per arm is eight $(N=8)$ and the reference signal can range in the interval $[-1,1]$. The yellow areas are those in which a single SM of a set can be activated in the upper arm. Fig. 4(a) shows the case of using a single sensor per arm. In this case, a single SM of the upper arm will be activated only when the reference signal is located within the two upper zones. Since the reference signal will only reach those zones when operating with high modulation indices $\left(m_{a}>0.5\right.$ in this example), measurements can not be performed for the full operation range of the converter. One the other hand, Fig. 4(b)

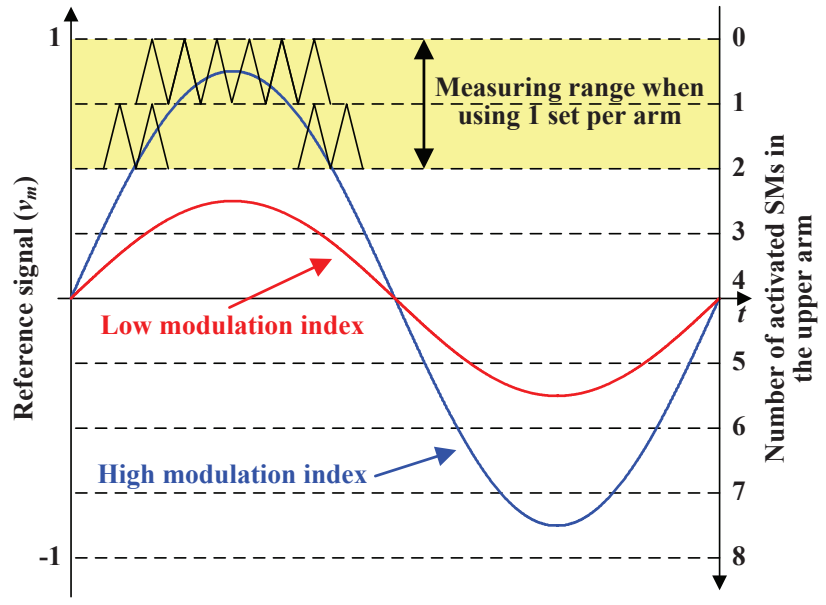

(a)

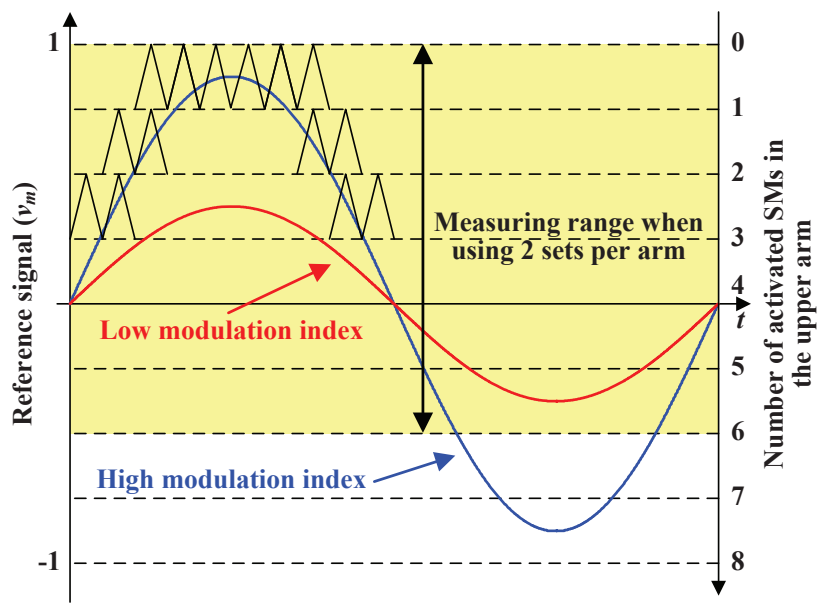

(b)

Fig. 4. Measuring range example for the upper arm with $N=8$ when using: (a) one and (b) two measuring sets per arm.

shows the case of using two voltage sensors per arm. Now, the yellow areas where a single SM in each set may be activated involve both low and large modulation indices. Consequently, capacitor voltage measurements can be performed for the full range of operation of the converter.

\section{E. Practical Implementation}

In high-voltage applications, where the number of SMs per arm is very high, different aspects have to be considered to select the proper number of voltage sensors. The main criterion that should be optimized is the actual measuring frequency of the capacitor voltages. The sampling frequency of the voltage sensor is always twice the switching frequency. However, actual measures are performed when only one SM in the measuring set is active, and only one SM is measured at a time. For this reason, the higher is the number of SMs in a measuring set, the lower is the actual measuring frequency. This measuring frequency depends on several factors, such as the number of SMs per measuring set, the switching frequency, the modulation index, the voltage balancing algorithm, etc., it is therefore difficult to adjust and thus it is adjusted empirically. 


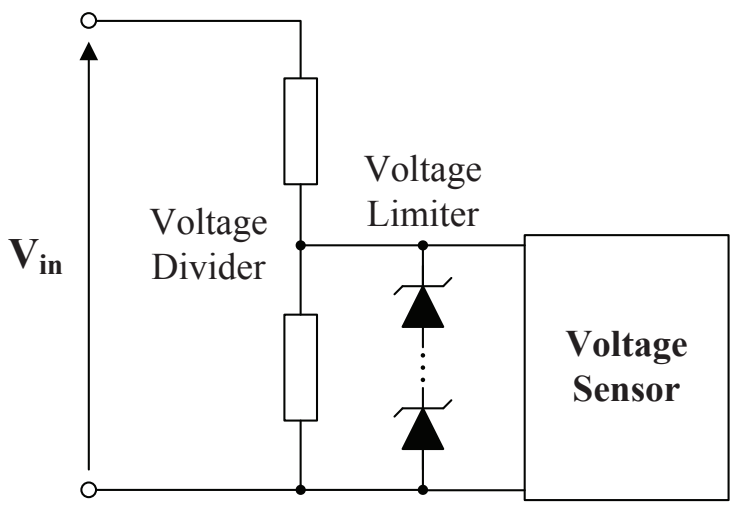

Fig. 5. Circuit diagram of the voltage sensor conditioning stage.

For example, in an MMC with 200 SMs per arm, 20 voltage sensors per arm can be used, which corresponds to one sensor for each $10 \mathrm{SMs}$. This means a significant reduction in the cost and complexity of the measuring system.

There is a second criterion that must be taken into account when defining the number of voltage sensors: the maximum voltage range of the sensor. The voltage sensors used in the proposed measuring technique have to withstand voltage values much larger than their usual measuring value, i.e., the voltage of a single capacitor. As the maximum voltage range is proportional to the number of SMs in a measuring set, this parameter has to be considered when defining the number of voltage sensors.

The requirement of withstanding a maximum voltage range much higher than the usual measuring value leads to an important reduction on the accuracy of the measurements. In order to solve this problem, a signal conditioning stage based on a voltage limiter is proposed. A schematic of the proposed solution is depicted in Fig. 5, where the measured voltage is reduced using a precision voltage divider made with resistors. The voltage seen by the voltage transducer is limited using a series of Zener diodes. This circuit reduces the maximum voltage applied to the sensor without compromising the measurement accuracy.

\section{Enforced Measuring Algorithm}

The proposed technique uses the favorable situations that happen in the operation of the MMC to perform the individual capacitor voltage measurement. However, it does not ensure measurement of all the SM capacitor voltages in a delimited time interval. For this reason, the measuring technique can be complemented by an enforced measuring algorithm. If a particular SM capacitor voltage is not measured for a certain time, the enforced measuring algorithm will facilitate the activation of that SM alone in the measuring set.

Unlike the measuring and estimation technique, the enforced measuring algorithm depends on the internal operation of the voltage balancing algorithm. In this paper, a voltage balancing algorithm based on sorting the SM capacitor voltages has been considered [26]. This algorithm also reduces the switching frequency of the power devices by activating or deactivating the minimum number of SMs whenever there is a change

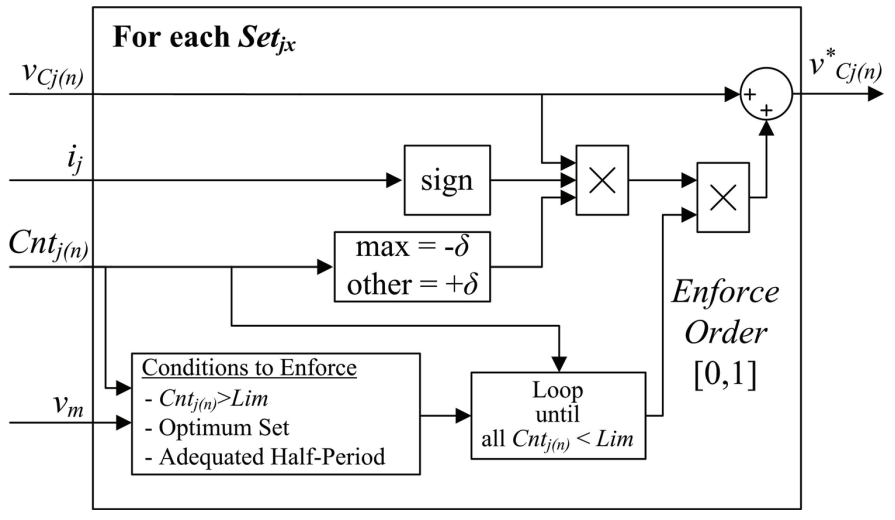

Fig. 6. Block diagram of the enforced measuring algorithm.

in the output voltage level. The switching reduction feature is not necessary for the operation of the enforced measuring algorithm, which would also work properly with other sorting techniques [9].

In order to implement the enforced algorithm, the time since the last single measurement of each SM $\left(C n t_{j(n)}\right)$ needs to be considered. If a particular time surpasses a pre-defined limit, the enforced measuring algorithm will be activated. In order not to interfere much with the operation of the converter, this algorithm will increase the probability of the SM to be activated alone in the measuring set by increasing its priority and decreasing the priority of the other SMs in the same measuring set. This is achieved by modifying the capacitor voltage values $v_{C j(n)}^{*}$ introduced in the voltage balancing algorithm. In the case that the direction of the arm current is such that it charges the SM capacitors, the estimated voltage of the target SM will be decreased by a certain percentage $\delta$. It is increased by the same amount if the arm current goes in the discharging direction. The priority reduction of the other SMs in the measuring set is achieved by proceeding in the opposite way, i.e., by increasing and decreasing their estimated voltage value a percentage $\delta$ when the direction of the arm current tends to charge or discharge their capacitors, respectively.

The percentage $\delta$ is designed to increase the priority of the SMs to be activated or deactivated. For a proper design, the SM capacitor voltage value that is increased or decreased by a percentage $\delta$ has to be higher or lower than all the other SMs, respectively. However, in order not to disturb excessively the voltage balancing operation, $\delta$ should increase the priority of the modified SM capacitor voltage value just a little over the maximum voltage of the SMs that are not modified. A good reference for the value of $\delta$ is to be twice the capacitor voltage ripple amplitude operating under rated conditions, i.e., if the voltage ripple amplitude is $5 \%$ of the reference value, $\delta$ should be set to about $10 \%$.

A block diagram showing the proposed enforcing algorithm is depicted in Fig. 6. In this figure, the algorithm of the priority modification process is detailed. The requirements for activating the enforced measuring algorithm are also summarized.

The first of these requirements is an excessive amount of time in which single measurements of an SM capacitor voltage have not been taken. However, as this algorithm modifies the 
TABLE I

SPECIFICATIONS OF THE SimUlation AND LABORATORY TEST CONVERTERS

\begin{tabular}{l|l|l}
\hline \hline Parameter & Simulation & Experimental \\
\hline Number of SMs per Arm, $N$ & 20 & 8 \\
\hline SM Capacitors, $C$ & $1500 \mu \mathrm{F}$ & $1500 \mu \mathrm{F}$ \\
\hline Arm Inductors, $L$ & $3 \mathrm{mH}$ & $3 \mathrm{mH}$ \\
\hline DC-Link Voltage, $V_{d c}$ & $40 \mathrm{kV}$ & $400 \mathrm{~V}$ \\
\hline Carrier Frequency, $f_{s w}$ & $5 \mathrm{kHz}$ & $1.724 \mathrm{kHz}$ \\
\hline Sampling Frequency, $f_{s a m p}$ & $100 \mathrm{kHz}$ & $34.482 \mathrm{kHz}$ \\
\hline Output Frequency, $f$ & $50 \mathrm{~Hz}$ & $50 \mathrm{~Hz}$ \\
\hline Grid Inductor, $L_{A C}$ & $12 \mathrm{mH}$ & - \\
\hline RMS Grid Voltage, $V_{A C}$ & $9.9 \mathrm{kV}$ & - \\
\hline RMS Load Current, $I_{a}$ & $200 \mathrm{~A}$ & - \\
\hline Load Current Phase Angle, $\varphi$ & $45^{\circ}$ & - \\
\hline Load Resistor, $R_{a}$ & - & $17 \Omega$ \\
\hline Load Inductor, $L_{a}$ & - & $6 \mathrm{mH}$ \\
\hline \hline
\end{tabular}

operation of the converter, more considerations have to be taken into account to minimize its negative effects. Performing single measurements in a particular arm can only happen during one half-period of the modulation signal and when the values are near zero, i.e., when the number of SMs activated in the arm is less than or equal to $N / 2+1$. For this reason, the enforced measuring algorithm will only be activated in the adequate half-period, i.e., when the modulation signal is positive for the upper arm and negative for the lower arm.

A second restriction that has to be considered in the application of the enforced measuring algorithm is that more than one SM can surpass the real measuring time limit at the same time. As only one SM can be enforced at a time, an order between them has to be imposed. A measuring priority could be defined by taking the times from the last single measurement and sorting them from the maximum to the minimum, but this may interfere significantly with the voltage balancing process, as explained next.

When enforcing a measurement, some time is required for changing the activated SMs between two different measuring sets. That is, if a particular SM capacitor in a measuring set needs to be measured and other SMs in the same set are activated, they have to be deactivated and the same amount need to be activated in other measuring sets. When using a voltage balancing algorithm with reduced switching transitions, a limited number of SMs will change each switching period and therefore several switching periods are needed until a voltage measurement can be performed. Let's assume that three capacitor voltages need to be measured because they have exceeded the time limit, and that the first SM is in the measuring set $S_{e t} t_{u 1}$, the second one is in the measuring set Set $_{u 2}$, and the third one is again in Set $_{u 1}$. Since the enforcing process will need some time to translate the activated SMs from one set to another, the enforced measuring process will start by focusing on the SMs that have surpassed the time limit in the same set before changing to another set. In this example, all the voltage measurements required in $\operatorname{Set}_{u 1}$ will be performed before proceeding with Set $_{u 2}$.
The enforced measuring algorithm modifies the values sent to the balancing algorithm in order to facilitate the activation of the desired SMs, but it does not force additional switching in the SMs. Moreover, when a voltage balancing algorithm with reduced switching frequency is used [25], [26], the switching frequency of the SMs is exactly the same. The unique negative effect produced by the enforced measuring algorithm is a slight increase in the capacitor voltage imbalances, as the voltage values seen by the balancing algorithm are different than the estimated/real ones.

\section{Simulation AND ExPERIMENTAl Results}

\section{A. Simulation Results}

The proposed measuring technique has been implemented in MATLAB/Simulink. Simulation studies have been performed using a switched model of a three-phase MMC connected to the grid through inductors $L_{A C}$. Nonideal acquisition effects have been modeled by adding random noise to both the voltage and current sensors. The results have been obtained for an MMC with twenty SMs per arm $(N=20)$ and where only two voltage sensors per arm are used. The specifications of this test converter are given in Table I.

A level-shifted PWM (LS-PWM) technique is implemented and the capacitor voltages are balanced using the algorithm proposed in [26]. A sinusoidal signal is generated as a voltage reference for the converter. A circulating current $\left(i_{\text {circ }}\right)$ controller is implemented to regulate the internal dynamics of the converter [27] besides reducing the capacitor voltage ripples. The controller is based on modifying the upper and lower arm modulation signals to regulate the circulating current. The reference of the modulation signal is calculated from the instantaneous values of the output current $\left(i_{a}\right)$ and modulation signal $\left(v_{m}\right)$, as follows:

$$
i_{\text {circ }}^{*}=\frac{i_{a} v_{m}}{2} .
$$

Fig. 7 presents the simulation results of the capacitor voltages of the upper arm SMs operating with a modulation index $m_{a}=0.7$. Two measuring techniques are compared; in Fig. 7(a) individual voltage sensors per SM are used while in Fig. 7(b) the proposed measuring technique is applied. Due to small differences between the estimated and the measured capacitor voltages, the capacitor voltages in Fig. 7(b) show more dispersion than those in Fig. 7(a), nevertheless such imbalances are small and within acceptable limits. Arm currents do not present any differences, demonstrating the effectiveness of the circulating current controller when using the proposed technique. It should be remarked that 40 voltage sensors per phase-leg would be required with the traditional measuring technique in this example, while our proposal requires only four sensors.

In order to demonstrate the effectiveness of the technique in correcting the accumulated errors, a simulation study using different SM capacitances has been performed. In this simulation, the capacitances have been modified randomly, following a normal distribution with a mean value of $1500 \mu \mathrm{F}$ and a variance of $10 \%$. Fig. 8 presents the simulation results when 

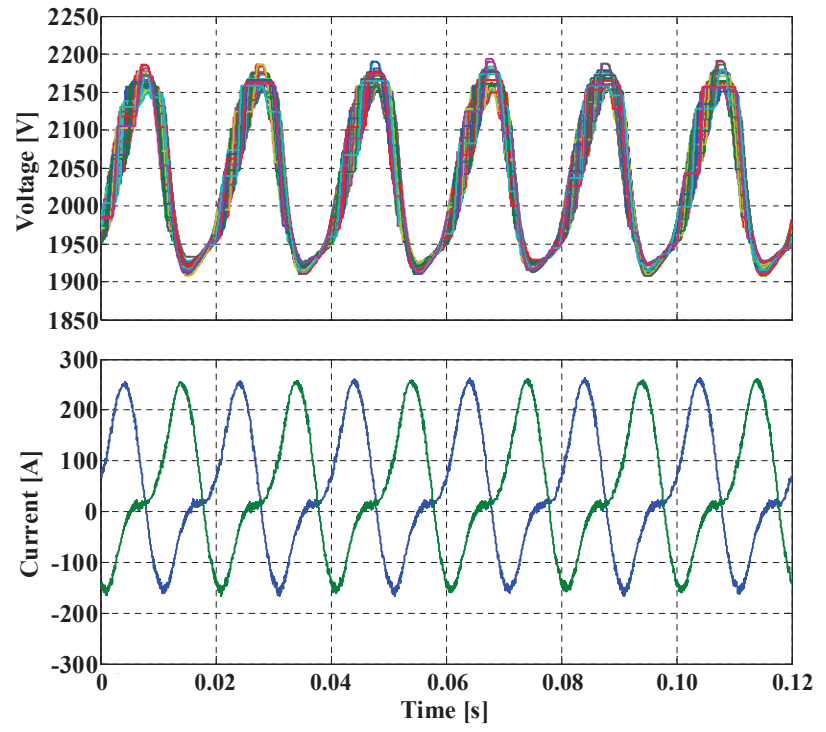

(a)

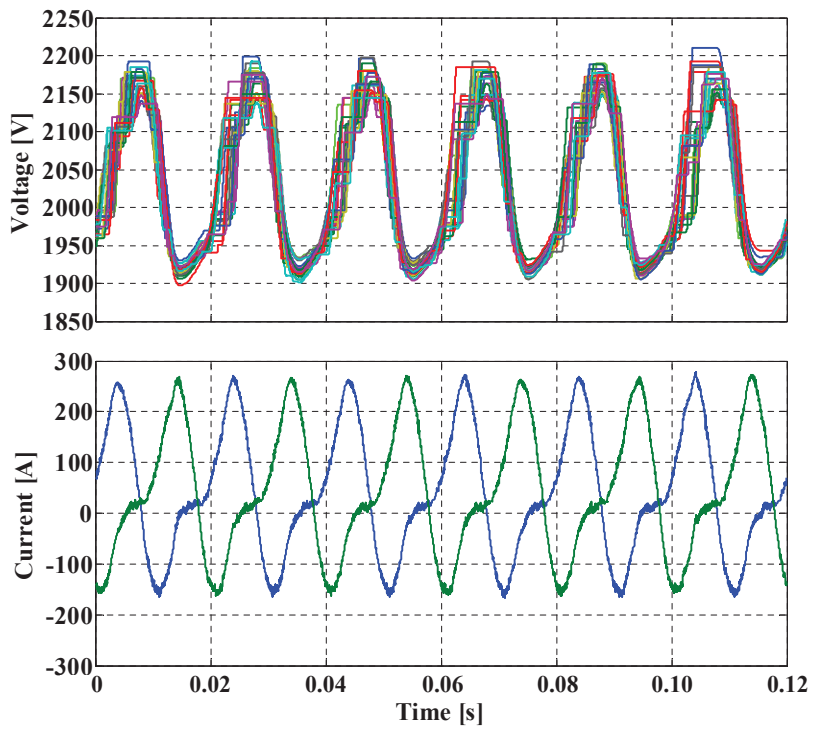

(b)

Fig. 7. Simulation results with $N=20$ and $m_{a}=0.7$. Upper arm capacitor voltages and arm currents with: (a) individual and (b) the proposed measuring techniques.

using the proposed technique in a converter with the same conditions than those in Fig. 7, but randomly modifying the capacitance values. Fig. 8(a) shows the capacitor voltages of the upper arm SMs and Fig. 8(b) shows the estimation errors (the estimated value minus the real value) of the capacitor voltages $v_{C a u 11}, v_{C a u 14}$, and $v_{C a u 15}$, which capacitances are $1745 \mu \mathrm{F}, 1500 \mu \mathrm{F}$ and $1227 \mu \mathrm{F}$, respectively. As it can be seen the errors are periodically corrected, although the capacitor voltages are less balanced and have increased voltage ripples. This is due to both, the intrinsic system dynamic and the updated information provided by actual measurements.

The periodic updating with actual values provides robustness to the estimation algorithm. However, if one or more capacitors have a capacitance significantly smaller than the nominal one, the proposed measuring system may produce

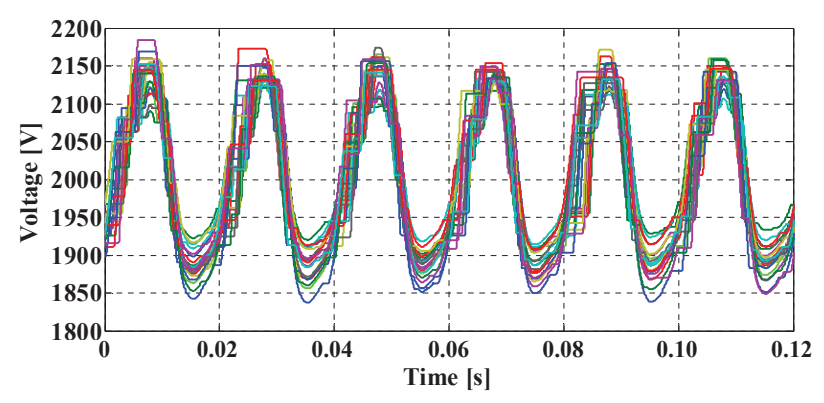

(a)

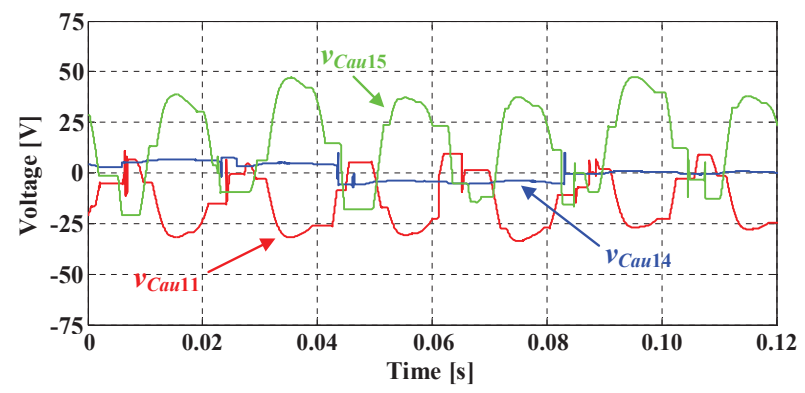

(b)

Fig. 8. Simulation results with the proposed measuring technique when considering different capacitances: (a) Upper arm capacitor voltages and (b) errors of the estimated capacitor voltages $v_{C a u 11}, v_{C a u 14}$, and $v_{C a u 15}$, which capacitances are $1745 \mu \mathrm{F}, 1500 \mu \mathrm{F}$, and $1227 \mu \mathrm{F}$, respectively.

instability to the converter. This can be demonstrated by a simplified analysis where the capacitor voltage ripples are neglected. Then, if a capacitor voltage was different than the reference value, the voltage balancing algorithm would activate or deactivate the corresponding SM until the estimated voltage equals the other capacitor voltages in the arm. However, if the real capacitance of the SM $\left(C_{\text {real }}\right)$ was smaller than the expected one $\left(C_{e s t}\right)$, i.e. the nominal capacitance, the change in the capacitor voltage $\left(\Delta v_{\text {real }}\right)$ would be larger than the estimated one $\left(\Delta v_{\text {est }}\right)$ :

$$
\Delta v_{\text {real }}=\Delta v_{e s t} \frac{C_{\text {est }}}{C_{\text {real }}} .
$$

If the actual capacitance was smaller than half the estimated capacitance $\left(C_{\text {real }}<C_{\text {est }} / 2\right)$, the variation of the capacitor voltage after compensation would be more than twice the estimated variation, and consequently the error of the voltage would be larger than the original one but in the opposite direction. The measuring system would realize of this in the next sampling and consequently the balancing controller will try to compensate for it. Then, the absolute value of the error would increase continuously making the system unstable. On the contrary, in the case that the SM capacitance was larger than half of the estimated one $\left(C_{\text {real }}>C_{\text {est }} / 2\right)$ the absolute value of the error after compensating would decrease and hence the system would be stable. It should be remarked that, in practice, having a capacitance smaller than $50 \%$ of its nominal value is not expected. Furthermore, the instability problem can be easily avoided by using an estimation algorithm that updates the capacitor values.

A single line-to-ground fault has been simulated to evaluate the proposed measuring technique performance during current 


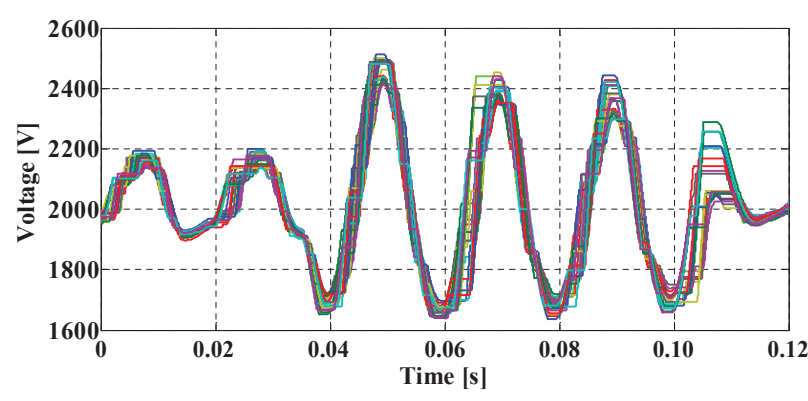

(a)

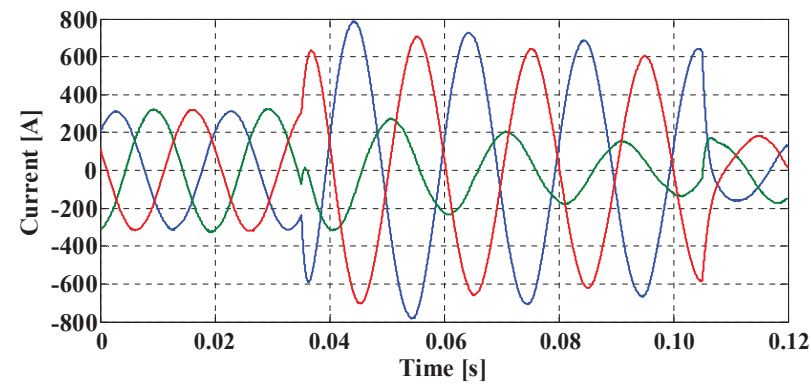

(b)

Fig. 9. Simulation results using the proposed measuring technique with a single line-to-ground fault: (a) Upper arm capacitor voltages and (b) output currents.

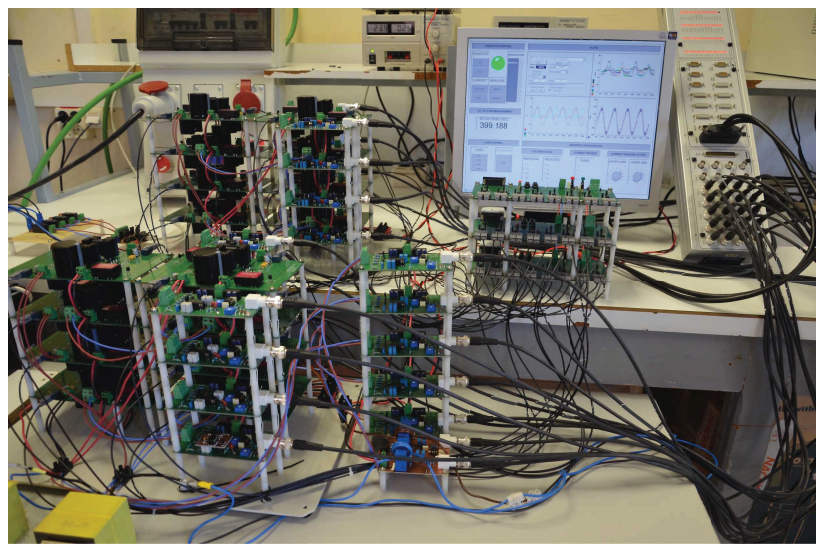

Fig. 10. MMC phase-leg laboratory prototype with eight SMs per arm $(N=$ 8).

transients. Fig. 9(a) show the upper arm capacitor voltages and Fig. 9(b) the output currents during a fault in Phase a, which has been applied from $t=0.035 \mathrm{~s}$ to $t=0.105 \mathrm{~s}$. Despite the current transients, the estimation method works properly, and the capacitor voltages are well balanced at all times.

\section{B. Experimental Results}

The proposed measuring technique has been implemented and tested in a low-power laboratory prototype of a phase-leg, with eight SMs per arm $(N=8)$ operating with an R-L load. The prototype has been implemented using silicon-carbide (SiC) technology, with MOSFET devices CREE CMF20120D and Schottky diodes CREE C4D10120D. Main control and acquisition have been performed using a dSPACE DS1103 platform and ControlDesk software. A photograph of the experimental prototype is presented in Fig. 10. Control and modulation of the converter is performed as explained in Subsection IV-A, and the main data of the prototype are given in Table I. Different static and dynamic tests have been performed in order to demonstrate the effectiveness of the proposed technique.

Figs. 11 and 12 present experimental results obtained with the individual measuring technique and the proposed technique, respectively. Both tests have been obtained working with a modulation index $m_{a}=0.9$. Figs. 11(a) and 12(a) show the upper arm capacitor voltages. As it can be seen, the voltages in Fig 12(a) with the proposed technique present a slightly higher dispersion than those in Fig 11(a), but without increasing significantly the capacitor voltage ripple amplitudes. Figs. 11(b) and 12(b)present the output voltages of the converter, while Figs. 11(c) and 12(c) depict the output current. No significant differences can be appreciated in the waveforms between using the individual and the proposed measuring techniques. Therefore, it can be stated that the performance of the MMC with the proposed technique is very similar to using the individual measuring technique.

The effectiveness of the proposed technique has been evaluated by measuring the dispersion of the capacitor voltages. The parameter to quantify such dispersion is the standard deviation, which is calculated for each instant of time, as follows:

$$
s_{N}=\sqrt{\frac{1}{N} \sum_{n=1}^{N}\left(v_{C j(n)}-\overline{v_{C j}}\right)^{2}},
$$

where $\overline{v_{C j}}$ is the average value of all the capacitor voltages in the arm at that instant of time. The instantaneous value of the standard deviation has been averaged by a moving window of one-period width.

The averaged standard deviation of the capacitor voltages obtained with the individual measuring technique and the proposed technique are depicted in Fig. 13. Although the deviation curve is slightly higher with the proposed technique, it is relatively close to the one using the individual measuring technique. The average value of the standard deviation for the whole time interval represented is $1.04 \mathrm{~V}$ for the individual measuring technique and 1.43 Vfor the proposed technique.

In order to test the dynamic response of the proposed technique, experimental results operating with load changes have been obtained and are represented in Fig. 14. In this figure, the resistive component of the R-L load changes from $68 \Omega$ to $17 \Omega$. Then, it returns to the initial value (68 $\Omega$ ). The circulating current controller explained in Section IV-A is implemented. Fig. 14(a) depicts the upper arm capacitor voltages. As it can be observed, the capacitor voltages are quickly balanced when the load resistance value is decreased. On the contrary, some more time is required to achieve voltage balance when increasing the load resistance value. This delay is due to a change in the dynamic of the voltage balancing algorithm and it has nothing to do with the proposed measuring technique. When increasing the load resistance value, the output current and the arm currents decrease, and therefore the capability of the voltage balancing dynamic is reduced accordingly. The arm currents and the output current during this process are shown in Figs. 14(b) and (c) respectively. 


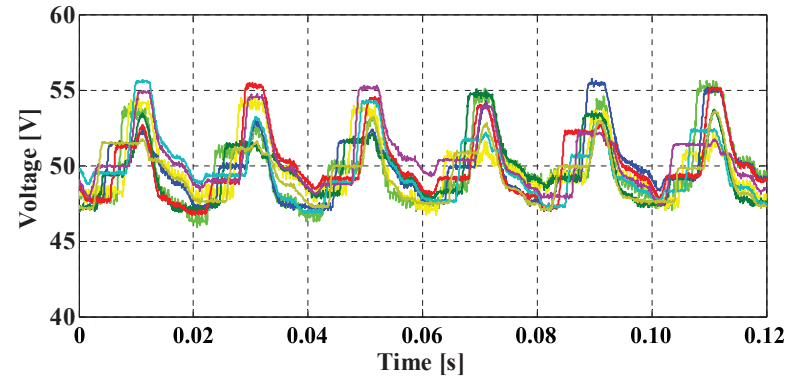

(a)

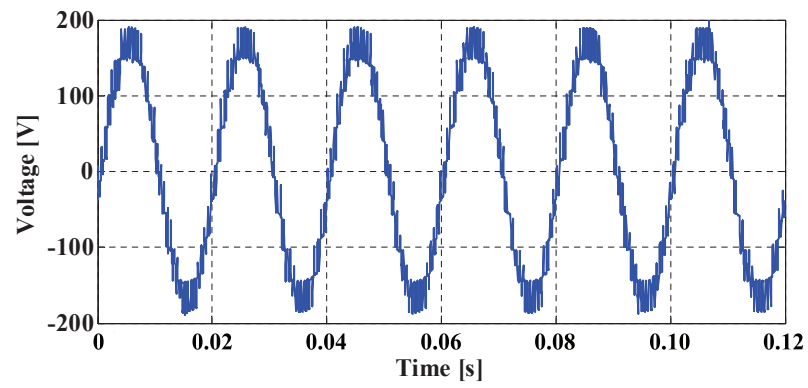

(b)

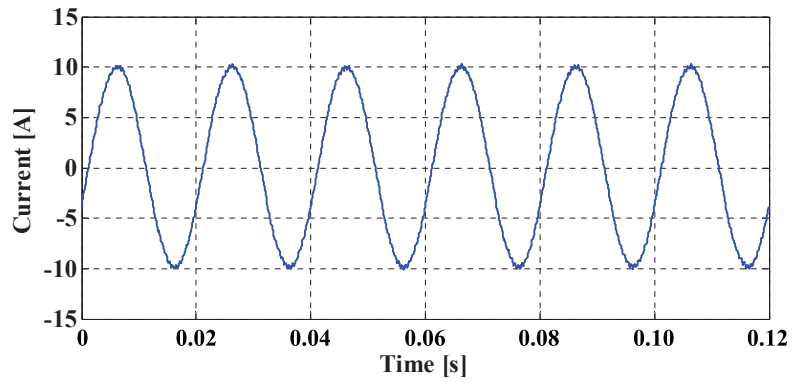

(c)

Fig. 11. Experimental results when using the individual measuring technique with $N=8$ and $m_{a}=0.9$ : (a) Upper arm capacitor voltages, (b) output voltage, and (c) output current.

\section{CONCLUSION}

In this paper, a new capacitor voltage measuring technique for MMCs has been presented. Each sensor is able to measure the capacitor voltages of multiple SMs. The arms of the MMC are divided into sets of SMs connected in series, and each sensor measures the output voltage of one of these sets. Two sensors per arm is the minimum number required; therefore, the total number of sensors is drastically reduced, and thus also the complexity and cost of the data acquisition system. The measuring technique involves capacitor voltage estimators that are updated whenever there is an actual measurement. This is performed when only one SM in the measuring set is activated. Despite the simplicity of the estimator, the fact that the voltage values are periodically updated limits the maximum accumulated error. The technique is complemented with an enforced measuring algorithm to ensure periodical refreshment of all the capacitor voltages. Simulation and experimental results demonstrate the effectiveness of the proposed technique. The capacitor voltage ripples increase slightly, but without compromising proper operation of the converter. This technique is especially interesting for MMCs with a large number of

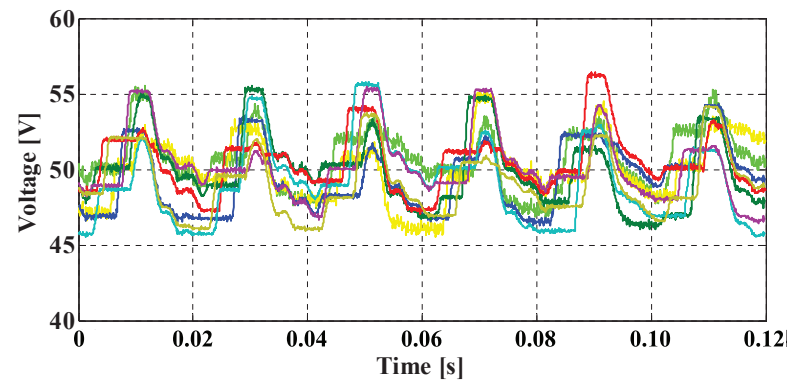

(a)

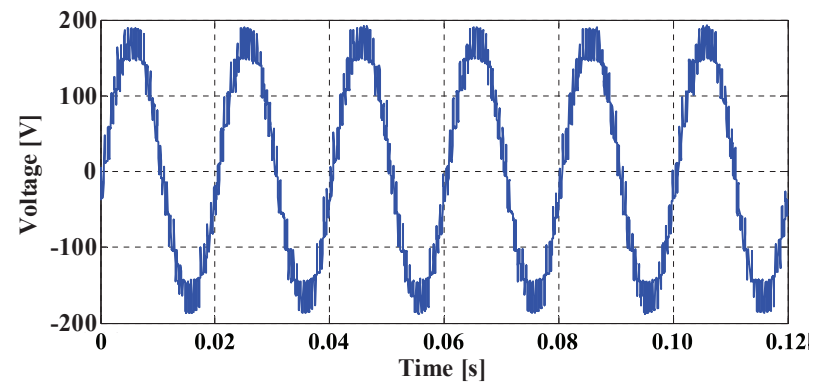

(b)

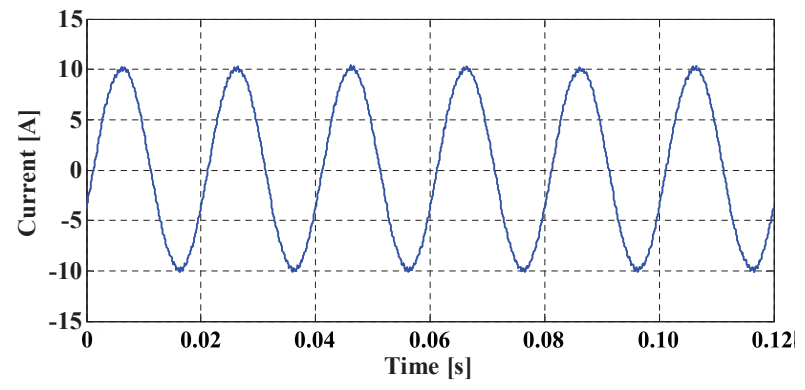

(c)

Fig. 12. Experimental results when using the proposed measuring technique with $N=8$ and $m_{a}=0.9$ : (a) Upper arm capacitor voltages, (b) output voltage, and (c) output current.

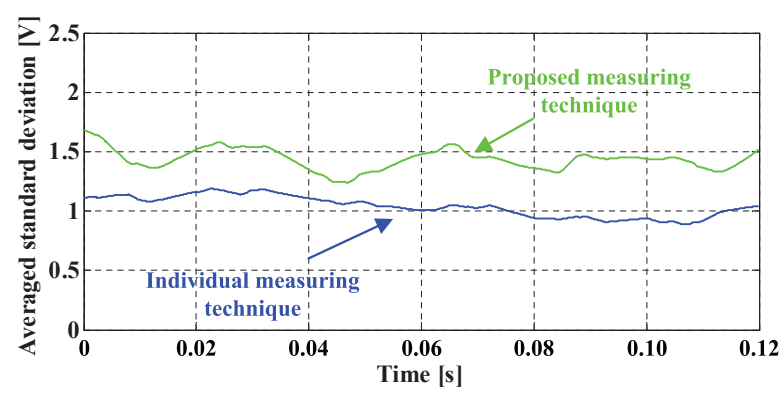

Fig. 13. Averaged standard deviation of the upper arm capacitor voltages obtained from experimental results with $m_{a}=0.9$.

SMs, due to the significantly lower number of voltage sensors required.

\section{REFERENCES}

[1] L.G. Franquelo, J. Rodriguez, J.I. Leon, S. Kouro, R. Portillo, and M.A.M. Prats, "The age of multilevel converters arrives," IEEE Ind. Electron. Magazine, vol. 2, no. 2, pp. 28-39, Jun. 2008.

[2] S. Kouro, M. Malinowski, K. Gopakumar, J. Pou, L.G. Franquelo, B. Wu, J. Rodriguez, M.A. Perez, and J.I. Leon, "Recent advances 


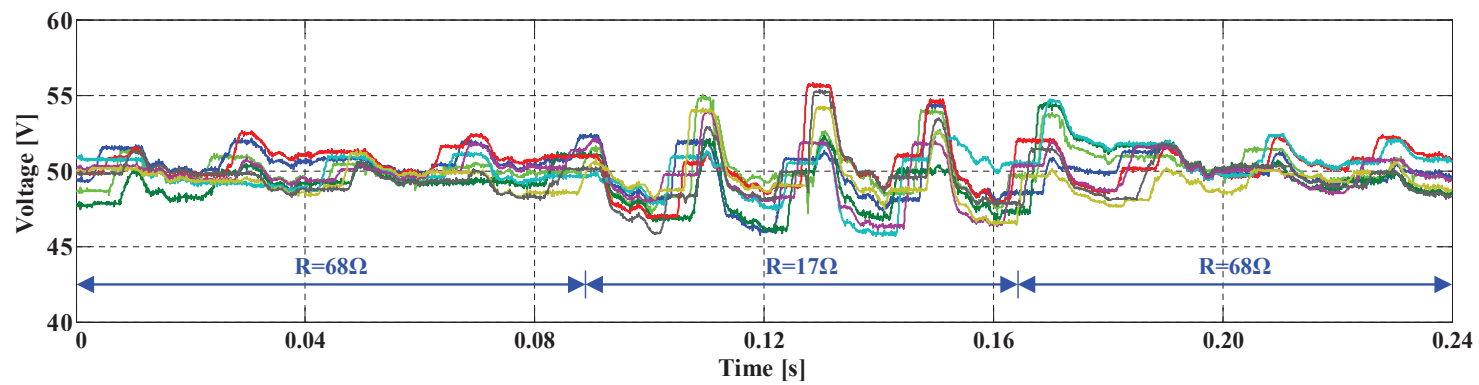

(a)

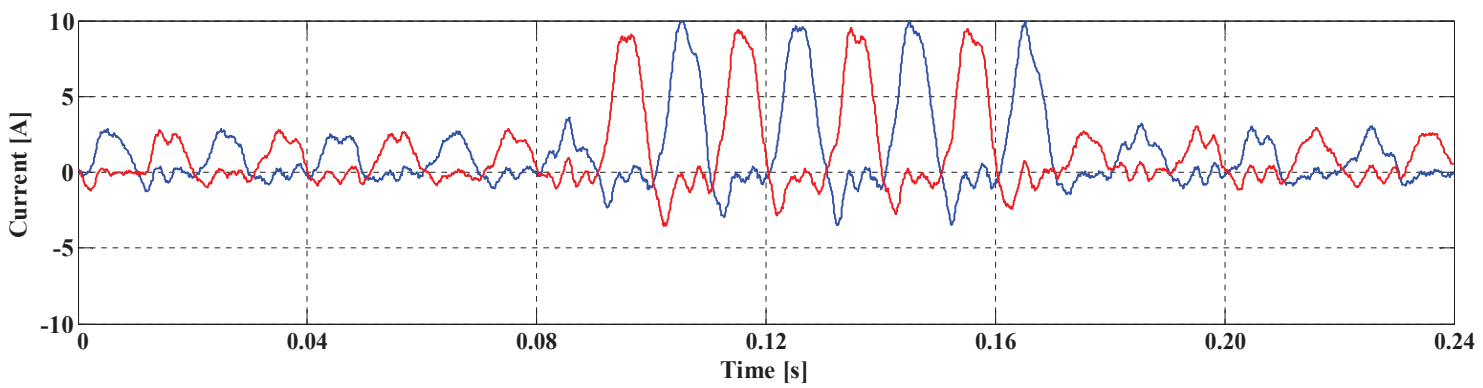

(b)

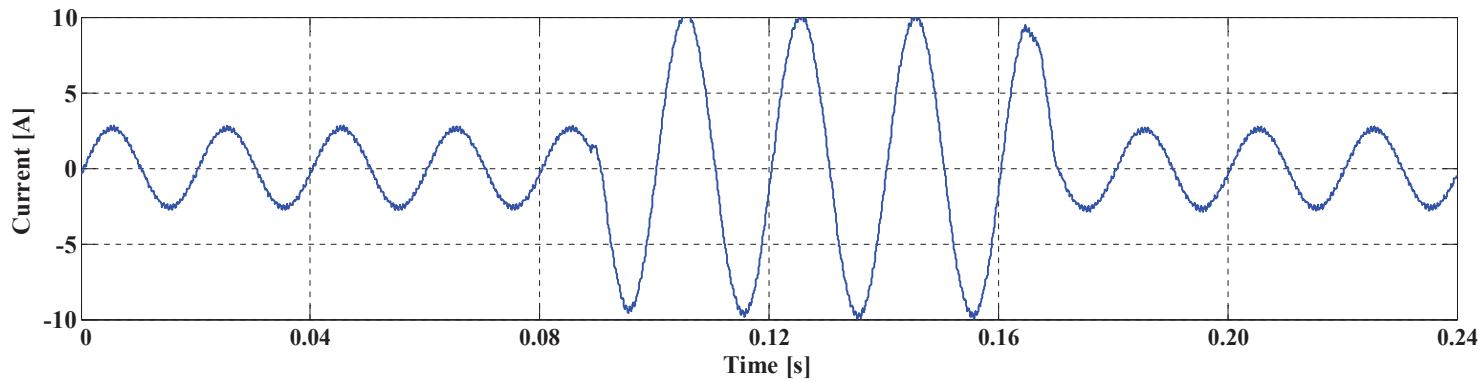

(c)

Fig. 14. Experimental results using the proposed measuring technique with load changes: (a) Upper arm capacitor voltages, (b) upper and lower arm currents, and (c) output current.

and industrial applications of multilevel converters," IEEE Trans. Ind. Electron., vol. 57, no. 8, pp. 2553-2580, Aug. 2010.

[3] A. Lesnicar and R. Marquardt, "An innovative modular multilevel converter topology suitable for a wide power range," in Proc. IEEE Bologna PowerTech Conference, Bologna, Italy, 23-26 Jun. 2003.

[4] A. Lesnicar and R. Marquardt, "A new modular voltage source inverter topology," in Proc. EPE, 2-4 Set. 2003, Toulouse, France.

[5] M. Hagiwara and H. Akagi, "Control and experiment of pulsewidthmodulated modular multilevel converters," IEEE Trans. Power Electron., vol. 24, no. 7, pp. 1737-1749, Jul. 2009.

[6] S. Debnath, J. Qin, B. Bahrani, M. Saeedifard, and P. Barbosa, ”Operation, control, and applications of the modular multilevel converter: a review," IEEE Trans. Power Electron., vol. 30, no. 1, pp. 37-53, Jan. 2015.

[7] M.A. Perez, S. Bernet, J. Rodriguez, S. Kouro, and R. Lizana, "Circuit topologies, modelling, control schemes and applications of modular multilevel converters," IEEE Trans. Power Electron., vol. 30, no. 1, pp. 4-17, Jan. 2015.

[8] K. Friedrich, "Modern HVDC PLUS application of VSC in modular multilevel converter topology," in Proc. IEEE International Symposium on Industrial Electronics (ISIE), 4-7 Jul. 2010, Bari, Italy, pp. 38073810.

[9] M. Saeedifard and R. Iravani, "Dynamic performance of a modular multilevel back-to-back HVDC system," IEEE Trans. Power Del., vol. 25, no. 4, pp. 2903-2912, Oct. 2010.

[10] H. Mohammadi P. and M. Tavakoli Bina, "A transformerless mediumvoltage STATCOM topology based on extended modular multilevel converters," IEEE Trans. Power Electron., vol. 26, no. 5, pp. 1534-1545, May 2011.
[11] S. Fan, K. Zhang, J. Xiong, and Y. Xue, "An improved control system for modular multilevel converters with new modulation strategy and voltage balancing control," IEEE Trans. Power Electron., vol. 30, no. 1, pp. 358371, Jan. 2015.

[12] F. Deng and Z. Chen, "A control method for voltage balancing in modular multilevel converters," IEEE Trans. Power Electron., vol. 29, no. 1, pp. 66-76, Jan. 2014.

[13] G.S. Konstantinou and V.G. Agelidis, "Performance evaluation of halfbridge cascaded multilevel converters operated with multicarrier sinusoidal PWM techniques," in IEEE Conf. Ind. Electron. and Applicat. (ICIEA), 25-27 May 2009, Xi' an, China, pp. 3399-3404.

[14] K.H. Ahmed and G.P. Adam, "New modified staircase modulation and capacitor balancing strategy of 21-level modular multilevel converter for HVDC transmission systems," in IET International Conference on Power Electronics, Machines and Drives (PEMD), 8-10 Apr. 2014, Manchester, UK, pp. 1-6.

[15] K. Ilves, A. Antonopoulos, S. Norrga, and H.P. Nee, "A new modulation method for the modular multilevel converter allowing fundamental switching frequency," IEEE Trans. Power Electron., vol. 27, no. 8, pp. 3482-3494, Aug. 2012.

[16] H. Nademi, A. Das, and L. Norum, "Modular multilevel converter with an adaptive observer of capacitor voltages," IEEE Trans. Power Electron., vol. 30, no. 1, pp. 235-248, Jan. 2015.

[17] S. D'Arco and J.A. Suul, "Estimation of sub-module capacitor voltages in modular multilevel converters," in Proc. European Conference on Power Electronics ans Applications (EPE), 2-6 Sept. 2013, Lille, France, pp. 1-10.

[18] J. Mei, K. Shen, B. Xiao, and L.M. Tolbert, "A new selective loop bias mapping phase disposition PWM with dynamic voltage balance 
capability for modular multilevel converter," IEEE Trans. Ind. Electron., vol. 61, no. 2, pp. 798-807, Feb. 2014.

[19] Y. Zhou, D. Jiang, P. Hu, and J. Guo, "A prototype of modular multilevel converters," IEEE Trans. Power Electron., vol. 29, no. 7, pp. 3267-3278, Jul. 2014.

[20] J.-J. Jung, H.-J. Lee, J.-I. Ha, and S.-K. Sul, "Reduced sampling rate for cell voltage sensing in high-level modular multilevel converter," in Proc. IEEE International Conference on Industrial Technology (ICIT), Feb. 26 2014-Mar. 1 2014, Busan, Korea, pp. 336-341.

[21] S. Shao, P.W. Wheeler, J.C. Clare, and A.J. Watson, "Fault detection for modular multilevel converters based on sliding mode observer," IEEE Trans. Power Electron., vol. 28, no. 11, pp. 4867-4872, Nov. 2013.

[22] I.A. Gowaid, G.P. Adam, A.M. Massoud, S. Ahmed, D. Holliday, and B.W. Williams, "Quasi two-level operation of modular multilevel monverter for use in a high-power DC transformer with DC fault isolation capability," IEEE Trans. Power Electron., vol. 30, no. 1, pp. 108-123, Jan. 2015.

[23] X. Shi, Z. Wang, B. Liu, Y. Liu, L.M. Tolbert, and F. Wang, "Characteristic investigation and control of a modular multilevel converterbased HVDC system under single-line-to-ground fault conditions," IEEE Trans. Power Electron., vol. 30, no. 1, pp. 408-421, Jan. 2015.

[24] Z. Rong, X. Lie, Y. Liangzhong, and B.W. Williams, "Design and operation of a hybrid modular multilevel converter," IEEE Trans. Power Electron., vol. 30, no. 3, pp. 1137-1146, Mar. 2015.

[25] Q. Tu, Z. Xu, and L. Xu, "Reduced-switching frequency modulation and circulating current suppression for modular multilevel converters," IEEE Tran. Power Del., vol. 26, no. 3, pp. 2009-2017, Jul. 2011.

[26] R. Darus, J. Pou, G. Konstantinou, S. Ceballos, R. Picas, and V.G. Agelidis, "A modified voltage balancing algorithm for the modular multilevel converter: evaluation for staircase and phase-disposition PWM," IEEE Trans. Power Electron., vol. 30, no. 8, pp. 4119-4127, Aug. 2015.

[27] J. Pou, S. Ceballos, G. Konstantinou, V.G. Agelidis, R. Picas, and J. Zaragoza, "Circulating current injection methods based on instantaneous information for the modular multilevel converter," IEEE Trans. Ind. Electron., vol. 62, no. 2, pp. 777-788, Feb. 2015.

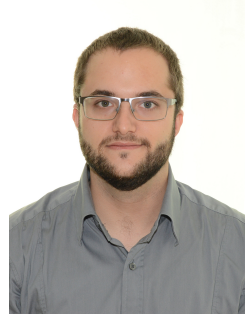

Ricard Picas (S'12) received the B.S. degree in electronic engineering and the M.S. degree in automatics and industrial electronics engineering from the Technical University of Catalonia (UPC), Catalonia, Spain, in 2010 and 2012, respectively, where he is currently working toward the Ph.D. degree.

Since 2012, he is working with the Terrassa Industrial Electronics Group, UPC as a Ph.D. student. His main research interests include multilevel converters and HVDC systems.

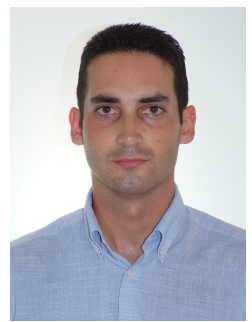

Jordi Zaragoza (S'08-M'12) received the B.S. degree in electronic engineering, the M.S. degree in automatic and electronic industrial engineering, and the Ph.D. degree from the Technical University of Catalonia (UPC), Catalonia, Spain, in 2001, 2004, and 2011, respectively.

In 2003, he joined the faculty of UPC as an Assistant Professor, where he became an Associate Professor in 2012. From September 2006 to September 2007, he was a Researcher at the Energy Unit of ROBOTIKER-TECNALIA Technologic Corporation, Basque Country, Spain. He is the author of more than 50 published technical papers and has been involved in several projects in the fields of power electronics and systems. His research interests include the modeling and control of power converters, multilevel converters, wind energy, power quality, and HVDC transmission systems.

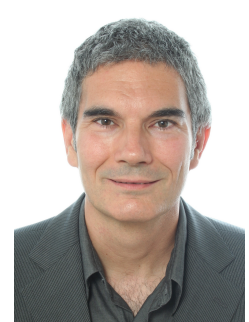

Josep Pou (S'97-M'03-SM'13) received the B.S., M.S., and Ph.D. degrees in electrical engineering from the Technical University of Catalonia (UPC), Catalonia, Spain, in 1989, 1996, and 2002, respectively.

In 1990, he joined the faculty of UPC as an Assistant Professor, where he became an Associate Professor in 1993. Since February 2013, he is a Professor with the University of New South Wales (UNSW), Sydney, Australia, on leave from the UPC, where he keeps a permanent position. From February 2001 to January 2002, and February 2005 to January 2006, he was a Researcher at the Center for Power Electronics Systems, Virginia Tech, Blacksburg, VA, USA. From January 2012 to January 2013, he was a Researcher at the Australian Energy Research Institute, UNSW. From 2006 to 2013, he collaborated with TECNALIA Research \& Innovation as a Research Consultant. He has authored more than 180 published technical papers and has been involved in several industrial projects and educational programs in the fields of power electronics and systems. His research interests include multilevel converters, renewable energy generation, energy storage, smart grids, and HVDC transmission systems.

Prof. Pou is a Member of the IEEE Industrial Electronics and the IEEE Power Electronics Societies.

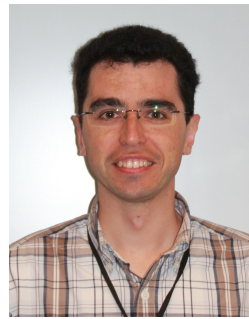

Salvador Ceballos received the M.S. degree in physics from the University of Cantabria, Santander, Spain, in 2001, and the M.S. and Ph.D. degrees in electronic engineering from the University of the Basque Country, Bilbao, Spain, in 2002 and 2008, respectively. Since 2002 he has been with Tecnalia Research and Innovation, Derio, Spain, where he is currently a Researcher in the Energy and Environmental Division. From May 2008 to May 2009 he was a Visiting Researcher at the Hydraulic and Maritime Research Centre, University College Cork (UCC), Cork, Ireland. From November 2014 to May 2015 he was a Visiting Researcher at the Australian Energy Research Institute, UNSW, Sydney, Australia. He has authored more than 100 published technical papers. His research interests include multilevel converters, fault-tolerant power electronic topologies, and renewable energy systems.

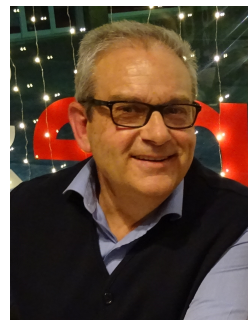

Josep Balcells (M'95-SM'06) was born in Vilarodona, Spain, in 1949, received the Master Engineering degree in 1975 and the Ph.D degree in 1983 from Universitat Politécnica de Catalunya (UPC). From 1975 to 1986 he was associated professor and since 1986 he is professor at Electronics Engineering Dept. in UPC and Technical consultant of CIRCUTOR SA. From 1976 to 1986 he was the head of R\&D in Power Electronics in AGUT SA (now a GE group company). His topics of interest are: Power Electronics, EMC in power systems, EMI measurement and filtering, design of EMC compliant power converters. Since 2005 he is Senior Member of IEEE. 